\title{
Morphometry Difference of the Hippocampal Formation Between Blind and Sighted Individuals
}

\author{
Ningning Pan ${ }^{1,2 t}$, Ke Zheng ${ }^{3 t}$, Yanna Zhao ${ }^{1}$, Dan Zhang ${ }^{4}$, Changxu Dong ${ }^{1}$, Junhai $X u^{3}$, \\ Xianglin Li5* and Yuanjie Zheng ${ }^{1 *}$ \\ ${ }^{1}$ School of Information Science and Engineering, Shandong Normal University, Jinan, China, ${ }^{2}$ Master of Public \\ Administration Education Center, Xinjiang Agricultural University, Xinjiang, China, ${ }^{3}$ College of Intelligence and Computing, \\ Tianjin Key Lab of Cognitive Computing and Application, Tianjin University, Tianjin, China, ${ }^{4}$ Department of Mathematics \\ and Computer Science, Eindhoven University of Technology, Eindhoven, Netherlands, ${ }^{5}$ Medical Imaging Research Institute, \\ Binzhou Medical University, Yantai, China
}

OPEN ACCESS

Edited by:

Lars Muckli,

University of Glasgow,

United Kingdom

Reviewed by:

Qiang Zheng,

Yantai University, China

Ning Mao,

Peking University People's Hospital,

China

Xiaodong Zhang,

Nankai University, China

${ }^{*}$ Correspondence:

Xianglin $\mathrm{Li}$

Xli163@163.com

Yuanjie Zheng

yjzheng@sdnu.edu.cn

${ }^{t}$ These authors have contributed equally to this work

Specialty section:

This article was submitted to Perception Science,

a section of the journal

Frontiers in Neuroscience

Received: 14 June 2021 Accepted: 07 October 2021 Published: 04 November 2021

Citation:

Pan N, Zheng K, Zhao Y, Zhang D,

Dong C, XU J, Li X and Zheng Y (2021) Morphometry Difference of the Hippocampal Formation Between

Blind and Sighted Individuals.

Front. Neurosci. 15:715749.

doi: 10.3389/fnins.2021.715749
The detailed morphometry alterations of the human hippocampal formation (HF) for blind individuals are still understudied. 50 subjects were recruited from Yantai Affiliated Hospital of Binzhou Medical University, including 16 congenital blindness, 14 late blindness, and 20 sighted controls. Volume and shape analysis were conducted between the blind (congenital or late) and sighted groups to observe the (sub)regional alterations of the HF. No significant difference of the hippocampal volume was observed between the blind and sighted subjects. Rightward asymmetry of the hippocampal volume was found for both congenital and late blind individuals, while no significant hemispheric difference was observed for the sighted controls. Shape analysis showed that the superior and inferior parts of both the hippocampal head and tail expanded, while the medial and lateral parts constrained for the blind individuals as compared to the sighted controls. The morphometry alterations for the congenital blind and late blind individuals are nearly the same. Significant expansion of the superior part of the hippocampal tail for both congenital and late blind groups were observed for the left hippocampi after FDR correction. Current results suggest that the cross-model plastic may occur in both hemispheres of the HF to improve the navigation ability without the stimuli of visual cues, and the alteration is more prominent for the left hemisphere.

Keywords: hippocampal formation, shape analysis, blindness, plasticity, spatial navigation

\section{INTRODUCTION}

Spatial navigation is a complex process which depends on the confluence of various surrounding information including vision, auditory, and haptic cues (Lackner and DiZio, 2005; Fortin et al., 2008). Among the sensory inputs that generate the final sense of orientation, vision possesses the ability to reunite plenty of spatial information presented simultaneously. The "place cell" in the hippocampal formation (HF) can deal with the proximal and distal space information and update the motion cues continuously, which will fire with the occurrence of visual cues (Muller and Kubie, 1987; Saleem et al., 2018). As the central node of mnemonic circuitry, the HF is involved in the visual-spatial memory function and plays a critical role in the processing of space orientation (O’Keefe et al., 1998; Eichenbaum et al., 1999; Alme et al., 2014). 
In contrast to the traditional studies that focus on the spatial navigation function of sighted individuals, structural alteration of human brain regions with the absence of visuals stimuli is particularly interesting (Gagnon et al., 2012). There is a pressing need for the blind individuals to develop alternative strategies that localize the surrounding objects using the remaining senses (Loomis et al., 2001; Giudice, 2018). It is interesting that congenitally blind subjects showed a better performance in the navigation tests which were conducted inside the life-size mazes or in the ecological environments than the blindfolded sighted individuals (Gagnon et al., 2010, 2012; Chebat et al., 2011). Stronger auditory sensitivity and navigation abilities were also found with early blind subjects as compared to the sighted controls (Dufour et al., 2005; Kupers and Ptito, 2014; Manescu et al., 2021). The $\mathrm{HF}$ is one of the regions that produce plastic changes (cross-modal plastic) in humans with the help of extensive visual-spatial navigation training (Lazzouni and Lepore, 2014; Chebat et al., 2018). Alterations in the right HF has been observed in early blind individuals using volume analysis method (Chebat et al., 2007). Both the frontal lobe and HF were activated during the learning process with the stimuli of auditory based on the studies using functional Magnetic Resonance Imaging (fMRI) data (Crottaz-Herbette et al., 2005; Naumer et al., 2009). Delineating the detailed morphometry changes of the HF is essential for the understanding of the plastic phenomenon during the navigation process without the stimuli of visual cues.

However, the HF is a complex brain region comprised of several substructures (Krogsrud et al., 2014; Romero et al., 2017). The volume of the whole HF could show no significant variation as increase or decrease of specific subfields may occur together (Fortin et al., 2008). Volumetric analysis used in previous studies thus might not be sensitive enough to detect detailed alterations of the HF between blind individuals and sighted controls. Shape analysis, on the other hand, has been performed to observe the local changes of brain regions in diverse communities (Lai et al., 2009; Shi et al., 2011; Tae et al., 2011; Ng et al., 2014; Gahm and Shi, 2016). Similar research was proposed in previous publications on the development of fetal brain regions including the HF and cerebellum (Ge et al., 2015; Xu et al., 2020). As to the blind hippocampi, relevant results demonstrated that the anterior part of the right HF is larger with blind individuals compared to the sighted controls based on shape analysis method (Leporé et al., 2009). On the contrary, significantly smaller regions was found in the posterior part of the right hippocampi, which indicates the heterogeneity of different subregions of the $\mathrm{HF}$ (Leporé et al., 2009). However, different patterns of dysfunction or different blinding timing (congenital and late blindness) were considered together in previous studies using shape analysis method, which may develop significant deviation of the HF from its normal surface among different onset patterns of blindness. The detailed morphometry alterations of the HF for either congenital or late blind individuals are still not well described.

In this study, 16, 14, and 20 of congenital blind, late blind, and sighted individuals were recruited, respectively, to calculate the morphometry difference of the HF between the blind and sighted individuals. Shape analysis based on Riemannian metric optimization on surfaces (RMOS) method was used to characterize the regional alterations of the HF that caused by blindness (Gahm and Shi, 2016). We hypothesized that current research may provide detailed representation of the morphometry alterations of the blind HF compared to that of the sighted controls. Different onset timing of blindness may exhibit diverse plastic patterns. Plastic changes may occur in both hemispheres of the blind HF, which may provide additional contributions for the understanding of cross-model plastic of human brain without the stimuli of visual cues.

\section{MATERIALS AND METHODS}

\section{Participants}

Thirty blind subjects (16 congenital blind and 14 late blind) and twenty sighted controls were recruited from Yantai Affiliated Hospital of Binzhou Medical University. The current cohort had been partly used to delineate the alteration of functional brain network in our previous publication (Li et al., 2019). The basic information of the current cohort is shown in Table 1. All blind subjects were diagnosed cataracts or retinal pigment degeneration based on the consensus of two professional ophthalmologists. No neurological disabilities except for the visual deprivation of the blindness were considered as one of the inclusion criteria in the current study. The approval from the Institutional Review Board of Binzhou Medical University was acquired for the conduction of current study. Written informed consent was obtained from each subject after understanding the purpose of this study.

\section{Data Acquisition}

T1 weighted MR images were acquired with a 3.0 T Siemens Skyra scanner at Yantai Affiliated Hospital of Binzhou Medical University. 3D MPRAGE sequence was used and the parameters are as follows: repetition time: $1,900 \mathrm{~ms}$, echo time: $2.52 \mathrm{~ms}$, inversion time: $1,100 \mathrm{~ms}$, voxel size: $1 \times 1 \times 1 \mathrm{~mm}^{3}$, matrix size: $256 \times 256$, flip angle: $90^{\circ}$.

\section{Segmentation of the Hippocampal Formation}

The HF was manually segmented by one anatomist with the help of ITK-SNAP software (Yushkevich et al., 2006), referenced by the previous studies and histological atlas (Ge et al., 2015; Yushkevich et al., 2015; Adler et al., 2018). Compared with fetal $\mathrm{HF}$, segmentation of the adult HF is easier and faster as the hippocampal tail is far away from corpus callosum and cingulate gyrus. The only challenge is the boundary between amygdala and hippocampal head as both of them are closely located in the temporal lobe (Konrad et al., 2009). The CSF between hippocampal head and amygdala was used for the boundary determination in the current segmentation protocol (Figure 1).

Twenty subjects were selected randomly from the whole cohort 3 months later and re-segmented manually by another anatomist to check the reliability of the above segmentation. Dice's coefficient was calculated based on the intraclass 
TABLE 1 | The demographic information of the cohort.

\begin{tabular}{|c|c|c|c|c|c|}
\hline Group & Con & Cong & $P$ value (Cong vs Con) & Late & $P$ value (Cong vs Con) \\
\hline Number (N) & 20 & 16 & 1 & 14 & 1 \\
\hline Age (year) & $21.97 \pm 1.16$ & $22.40 \pm 2.12$ & 0.43 & $22.18 \pm 1.67$ & 0.66 \\
\hline Gender (M/F) & $10 / 10$ & $12 / 4$ & 0.13 & $11 / 3$ & 0.09 \\
\hline Onset time (year) & $\backslash$ & 0 & $\backslash$ & $10.21 \pm 5.52$ & 1 \\
\hline
\end{tabular}

Two tailed student t-tests and Chi-square tests were conducted for comparison of age and gender distribution between blind and sighted groups, respectively. Con, control group; Cong, congenital blind group; Late, late blind group; M, male; F, female.

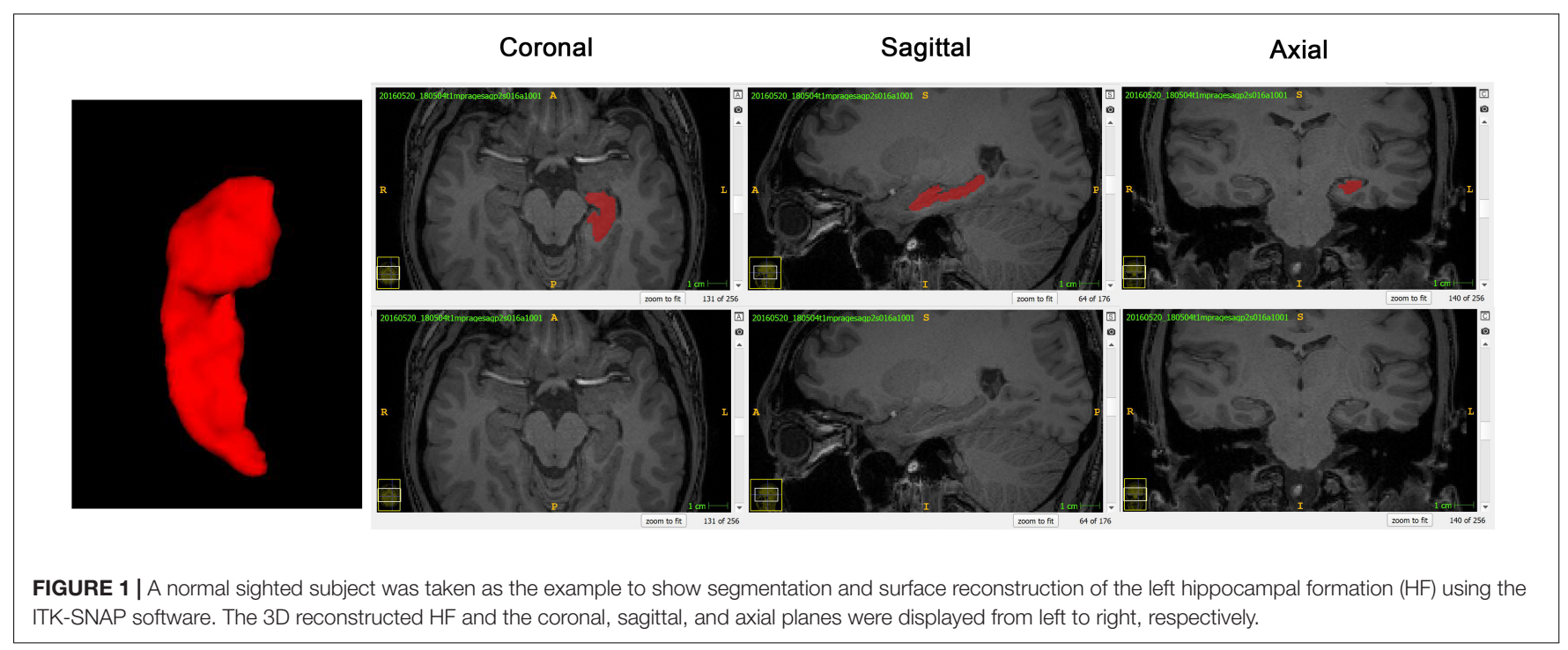

consistency of the two segmentations, which was also used in our previous publication (Ge et al., 2015).

\section{Volume Analysis}

Absolute volume of the HF was calculated based on the binary masks acquired from the segmentation section. The relative volume of the $\mathrm{HF}$ was calculated via the following formula: $\mathrm{V}_{\text {relative }}=\mathrm{V}_{\text {absolute }} / \mathrm{ICV}$, where the intracranial volume (ICV) for each subjects was acquired based on the Bet process results in FSL software (Jenkinson et al., 2012).

\section{Shape Analysis}

Prior to perform shape analysis, the hippocampal templates of both hemispheres were established separately using buildtemplateparelle.sh on ANTs software (Avants et al., 2009). Default options of ANTs software were selected for this process.

Surface meshes of the HF for all subjects as well as the template were then acquired from the binary masks. All surface meshes were re-meshed to 1,000 vertices based on the size of the HF as well as considering the computational cost. The individual remeshed surface was mapped to the re-meshed template based on RMOS algorithm (Gahm and Shi, 2016). Similar to the previous studies (Ge et al., 2015; Xu et al., 2020), the thickness of left and right hippocampi was measured separately and each vertex on the mapped surface was calculated by the distance from the surface vertex to the medial core of the HF. More detailed description of the shape analyses method can be found in our previous publication (Ge et al., 2015).

\section{Statistical Analysis}

Two tailed student $t$-test was conducted for the comparison of the hippocampal volume between the blind and sighted individuals, as well as the hemispheric difference within each group. The same $t$-test method was conducted on the thickness of the shape surface points to calculate the regional difference between the blind and sighted groups using Surfstat software on the MATLAB platform (Worsley et al., 2009). The volume and shape differences between the congenital/late blind individuals and sighted controls were calculated separately. The false discovery rate (FDR) correction was applied for the correction of multiple comparisons for all statistical tests. Significance for all statistical analysis was set to $p<0.05$.

\section{RESULTS}

\section{Study Cohort Characteristics and Reliability of the Segmentation}

Demographic and clinical characteristics of the study cohort are presented in Table 1. No significant difference of related characteristics (age and gender distribution) is found between the blind individuals (either congenital and late blindness) and sighted controls. 
The average Dice's coefficients are 0.9233 for the left hippocampi and 0.9352 for the right hemisphere, which demonstrate high reproducibility of the segmentation protocol in the current study.

\section{Volume Analysis}

Absolute and relative hippocampal volume information of the current cohort is shown in Table 2 and Figure 2. No significant difference of the hippocampal volumes (either absolute or relative volume) between blind (either congenital or late blind) and sighted subjects was found for both hemispheres $(p>0.05$, Table 2). Both congenital blind $(p=0.0079)$ and late blind $(p=0.0252)$ individuals show significantly rightward asymmetry of the absolute hippocampal volume, whereas control group does not show asymmetry difference $(p=0.2891)$.

\section{Shape Analysis \\ Congenital Blind Versus Control Group}

The statistical results ( $p$-value and $t$-value maps) that represent the comparison between congenital blind and control groups are shown in Figures 3, 4. Compared to the sighted control group, congenital blind individuals exhibit expansion of the shape surface mainly in the superior and inferior parts, while show contraction in the medial and lateral parts of both the

TABLE 2 | Volume information of the current cohort.

\begin{tabular}{|c|c|c|c|c|c|c|}
\hline & Hemisphere & Con & Cong & $P$ value (Cong vs Con) & Late & $P$ value (Late vs Con) \\
\hline \multirow[t]{2}{*}{ Absolute } & $L\left({ }^{*} 10^{3} \mathrm{~mm}^{3}\right)$ & $2.89 \pm 0.35$ & $2.70 \pm 0.38$ & 0.1195 & $2.85 \pm 0.34$ & 0.7262 \\
\hline & $\mathrm{R}\left({ }^{*} 10^{3} \mathrm{~mm}^{3}\right)$ & $2.92 \pm 0.39$ & $2.77 \pm 0.40$ & 0.2318 & $2.96 \pm 0.35$ & 0.8030 \\
\hline \multirow[t]{2}{*}{ Relative } & $L\left({ }^{\star} 10^{-3}\right)$ & $1.80 \pm 0.21$ & $1.70 \pm 0.19$ & 0.2406 & $1.70 \pm 0.17$ & 0.3847 \\
\hline & $R\left({ }^{*} 10^{-3}\right)$ & $1.80 \pm 0.22$ & $1.80 \pm 0.20$ & 0.4259 & $1.80 \pm 0.17$ & 0.8568 \\
\hline
\end{tabular}

L, left; $R$, right; Con, control group; Cong, congenital blind group; Late, late blind group. $p<0.05$ as statistically significant.

A

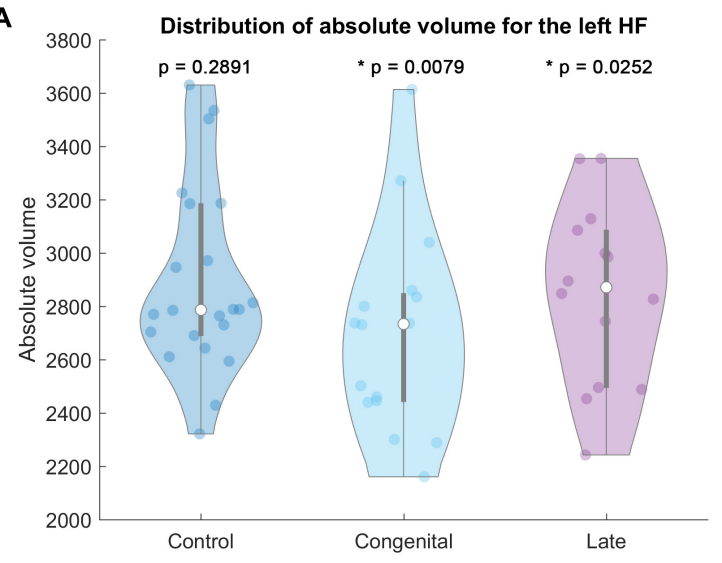

C

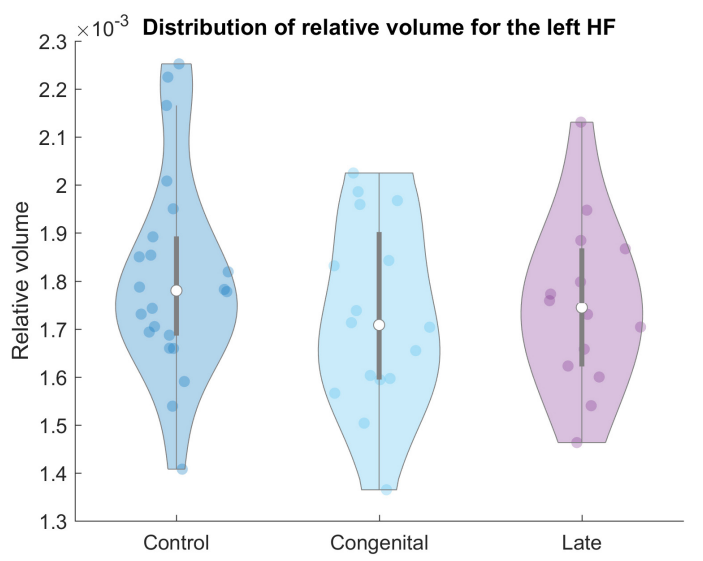

B

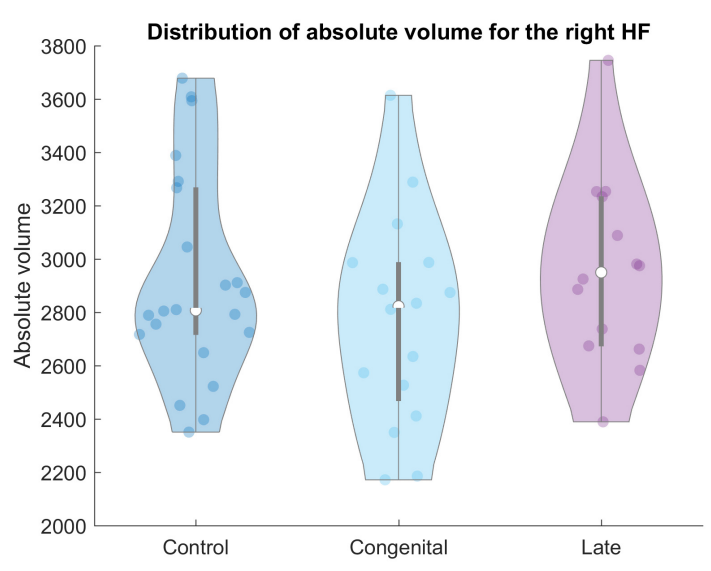

D

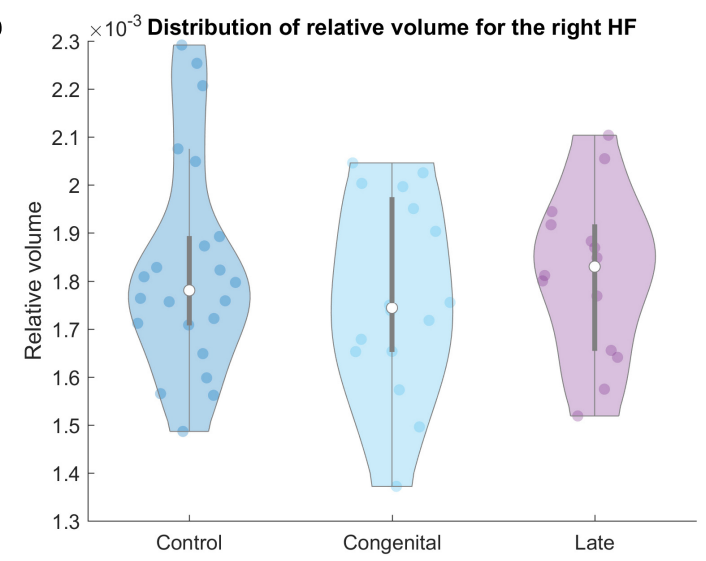

FIGURE 2 | Violin plot of the hippocampal volumes for each group. Top row absolute volume of the left (A) and right (B) HF. Bottom row relative volume of the left (C) and right (D) HF. $P$ values in the top left A part mean the hemispheric difference of the absolute volumes for each group, $p<0.05$ labeled with * as statistically significant. 


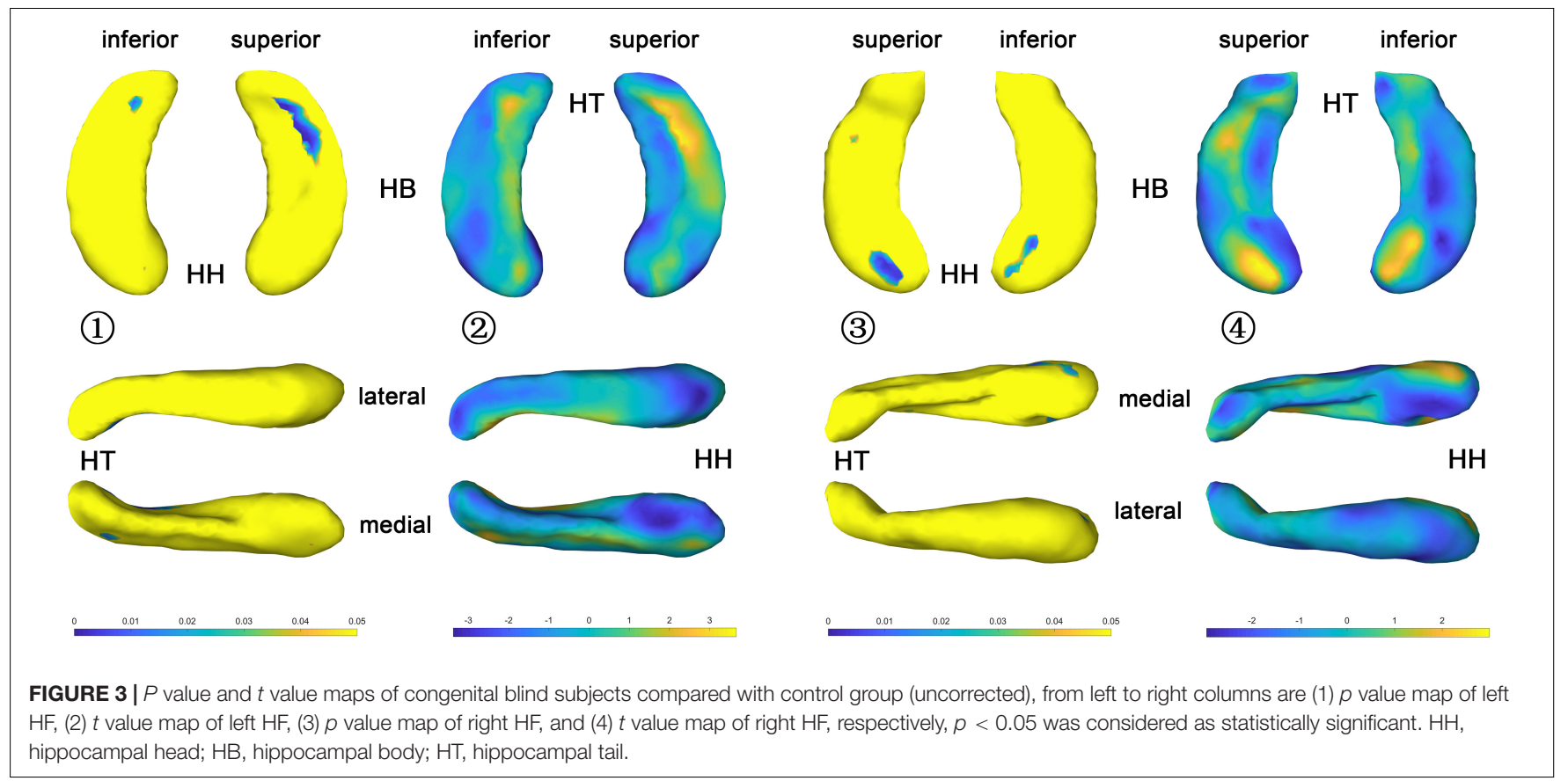

$\mathrm{HF}$, (2) $t$ value map of left $\mathrm{HF}$, (3) $p$ value map of right $\mathrm{HF}$, and (4) $t$ value map of right $\mathrm{HF}$, respectively, $p<0.05$ was considered as statistically significant. $\mathrm{HH}$, hippocampal head; HB, hippocampal body; HT, hippocampal tail.

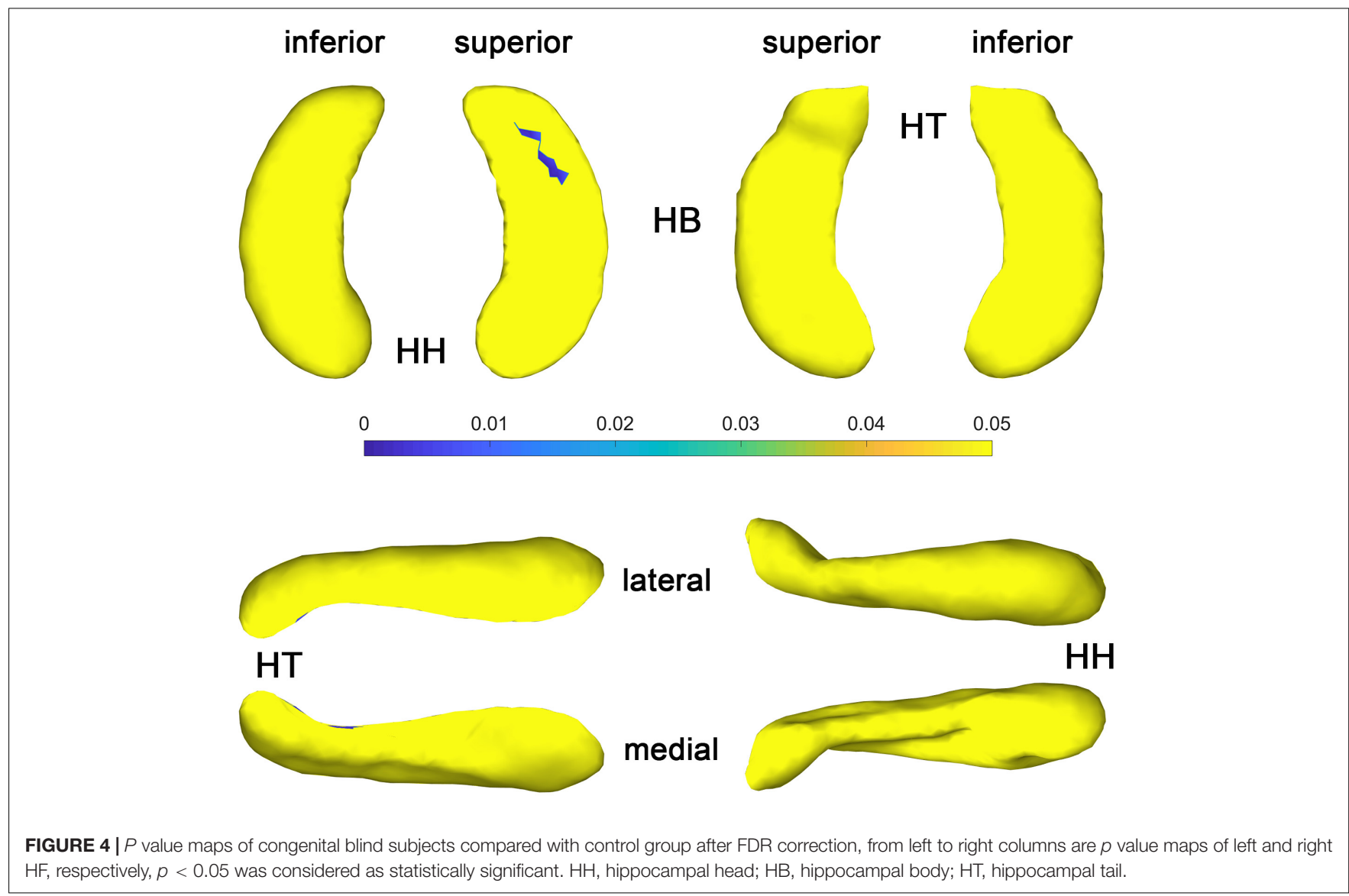

hippocampal head and tail for the left hippocampi (Figure 3-2). The increase or decrease of the thickness is not prominent in the region of hippocampal body. However, only the superior and inferior parts of the hippocampal tail expand significantly ( $p<0.05$, Figure 3-1). As to the right hippocampi, similar results are found from the $t$-value maps with the high thickness located 
in the superior and inferior parts of the hippocampal head and tail for the congenital blind group (Figure 3-4). The alteration of the hippocampal body is still not prominent for the right HF. Unlike the left hippocampi, the increased thickness of superior and inferior parts of the hippocampal head are statistically significant for the right hippocampi ( $p<0.05$, Figure 3-3). After FDR correction, the reserved regions that show significant alteration of the thickness between the congenital blind and sighted control groups only focus on the superior part of the hippocampal tail for the left hippocampi (Figure 4).

\section{Late Blind Versus Control Group}

The $t$-value maps that represent the difference of the hippocampal thickness between late blind and control groups and the $p$-value maps that show the significance of the difference are shown in Figure 5. Compared to the control group, late blind individuals show expansion in the superior and inferior parts of nearly the whole HF, while contraction appears in the medial and lateral parts of the hippocampal head for the left hippocampi (Figure 5-2). $P$-value maps show nearly the same result between the late blind and sighted control groups as compared to the comparison between the congenital blind and sighted control groups. Only the superior and inferior parts of the hippocampal tail of the left hippocampi are statistically significant (Figure 5-1). Small area of the anterior area of the hippocampal head also shows significant increase of the hippocampal thickness. For the right hippocampi, similar results are found from the $t$-value maps with the higher thickness located in the superior and inferior areas of both the hippocampal head and tail for the late blind group compared to the control group. The alteration of the hippocampal body is still not prominent for the right $\mathrm{HF}$ (Figure 5-4). Unlike the left hippocampi, the increased thickness of superior and inferior areas of the hippocampal head, and the superior part of the hippocampal tail are statistically significant for the right hippocampi (Figure 5-3). After FDR correction, the significant regions only focus on the superior part of the hippocampal tail and the most anterior part of the hippocampal head for the left hippocampi, whereas no significant different are found with the right hemisphere (Figure 6).

\section{DISCUSSION}

Volume analysis has been conducted in numerous studies to observe the morphometry changes of the HF (Woolard and Heckers, 2012; Yushkevich et al., 2015; Chen et al., 2018). Reduced volume was found with the right HF, while no significant difference was discovered with the left hemisphere of the congenital blind subjects compared to the sighted controls (Chebat et al., 2007). However, congenitally blind subjects performed better in the navigation tests that conducted inside the life-size mazes or in the ecological environments than the blindfolded sighted individuals (Gagnon et al., 2010, 2012; Chebat et al., 2011). When limiting the access to allothetic cues such as temperature and echolocation, blind subjects will lose their competitive advantages compared with controls in spatial navigation tasks (Gagnon et al., 2010). In addition, volume of the posterior $\mathrm{HF}$ is positively associated with the time of duration as the taxi drivers, while anterior HF shows negative correlation (Maguire et al., 2000). The posterior HF may expand significantly in response to store plenty of surrounding spatial information (Maguire et al., 2003). Moreover, different hemispheres of the cuneus was found significantly activated in distinct soundmediated pattern-spatial recognition tasks for blind subjects compared to the normal controls (Arno et al., 2001; Voss et al., 2008). The inferior parietal cortex can integrate the tactile inputs

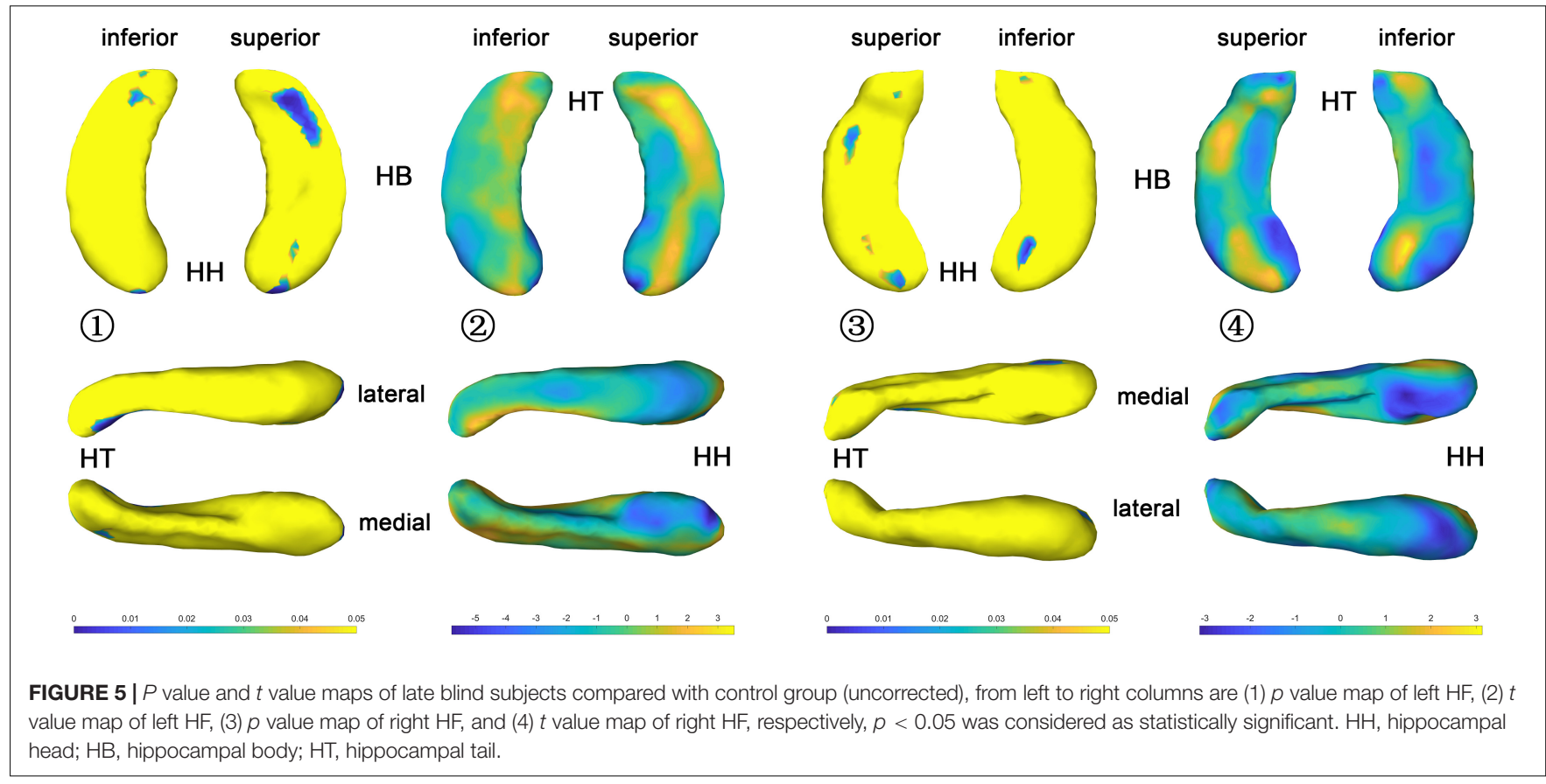




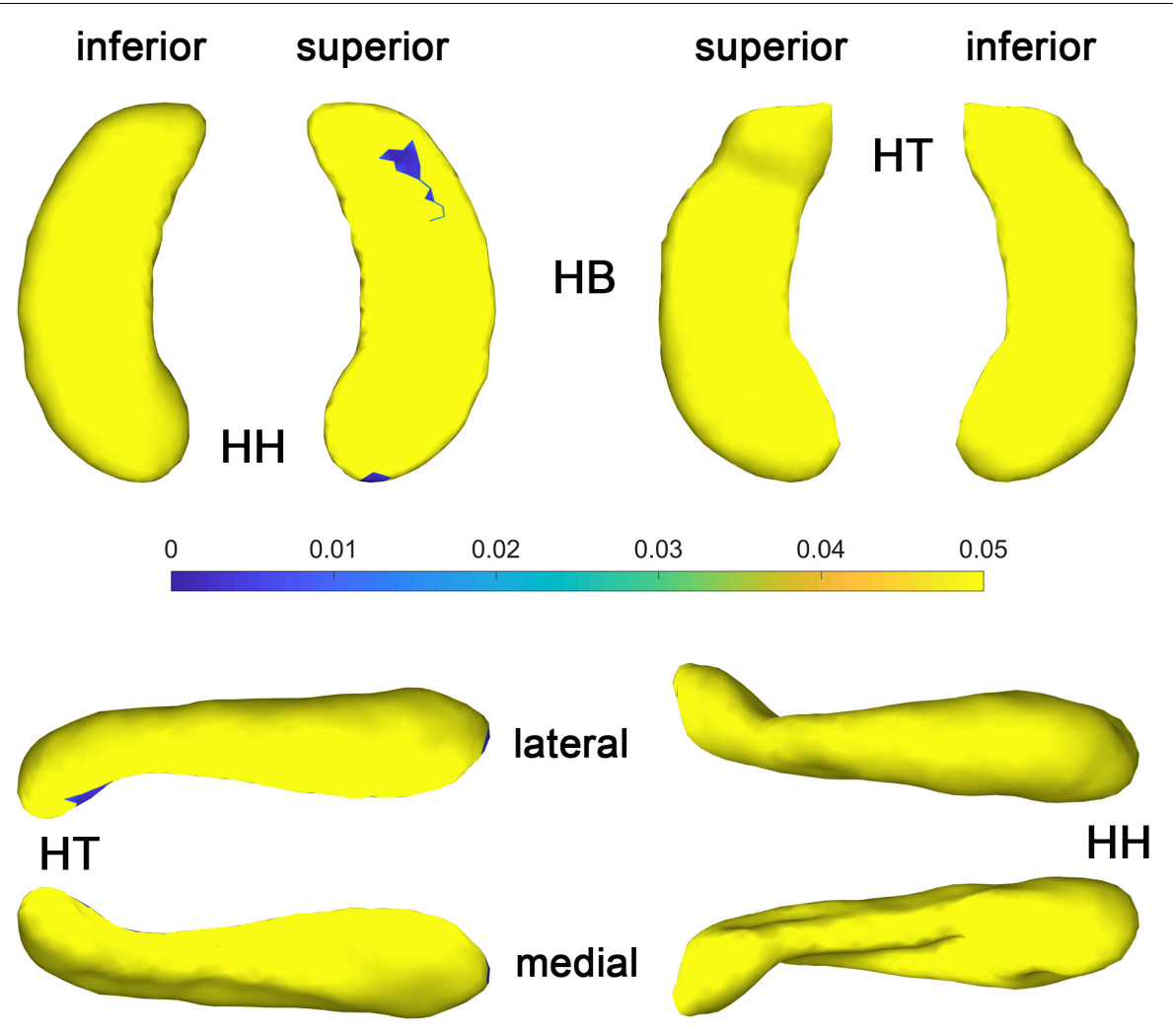

FIGURE 6 $\mid P$ value maps of late blind subjects compared with control group after FDR correction, from left to right columns are $p$ value maps of left and right $\mathrm{HF}$, respectively, $p<0.05$ was considered as statistically significant. $\mathrm{HH}$, hippocampal head; HB, hippocampal body; $\mathrm{HT}$, hippocampal tail.

to match visualization, auditory, and spatial information (Weeks et al., 2000; Collignon et al., 2007). Above studies indicated that the cross-modal plasticity or visual compensation may occur on the subregions of the HF as well as other brain regions to build effective spatial representations of the environment after the loss of visual stimuli.

However, recent research demonstrated no significant association between the volume of the $\mathrm{HF}$ and navigation function in the typical population (Weisberg et al., 2019). Similar results were also found in the current study with no significant difference between the blind (either congenital or late blindness) and sighted individuals of the hippocampal volume (either absolute or relative volume) (Table 2). Only rightward asymmetry of the hippocampal volume is found for the blind subjects, which indicates that the arisen of blindness may prompt the occurrence of hemispheric difference of the HF. Together with previous studies, there are debates of using the volumetric analysis to detect the morphometry changes of the blind HF. One possible reason is the volume analysis can only reflect the total representation of the brain (sub)regions, which is insufficient to delineate the subtle changes of the subfields of the relatively small regions such as the dentate gyrus, subiculum, and CA areas of the HF. However, the subfields of the HF are heterogeneous in function (Libby et al., 2012; Aanes et al., 2019). The increase of one subfield and the decrease of another one may occur simultaneously and lead to nearly no volumetric changes of the whole (sub)region. Another possibility is that the significantly correlation between the hippocampal volume and navigational ability is only detectable in extreme groups (Weisberg et al., 2019). It is hard to detect the differences of the ordinary blindness compared to the normal controls based on the volume analysis method. The delineation of the volume, on the other hand, may be influenced by the segmentation protocol, the resolution and field intensity of the MRI scanning, as well as the complexity of specific region that related to the research topics (Despotovic et al., 2015). The hippocampal volumes thus may be insufficient to be regarded as a biological marker for the supervision of navigation ability (Weisberg et al., 2019; Clark et al., 2020).

The shape analysis method that widely used recently is a more detailed morphometry analysis tool, which can be regarded as the powerful supplement to the traditional volume analysis method. Subtle changes of the small brain regions such as development of the fetal HF has been observed with the help of shape analysis method in the previous study (Ge et al., 2015). As the hippocampal surface is heterogeneous in function, distinct structural changes may occur in diverse hippocampal subfields with the arisen of blindness. Patterns of the hippocampal shapes of blind and sighted individuals have been described based on the conformal metric optimization on surfaces (CMOS) registration algorithm (Leporé et al., 2009). Compared to the control group, significantly increased thickness of the anterior part and decreased thickness of the posterior part of the right HP 
were found with blind individuals. On the contrary, no significant difference was observed for left HP between the blind and sighted controls (Leporé et al., 2009). However, the congenital and late blind subjects were considered together for the statistical analyses in the former study, which may introduce bias of the final conclusion. In the current research, we recruit congenital blind, late blind, and sighted normal control individuals to observe the volume and surface changes that caused by blindness. Compared to the sighted individuals, increased thickness of the superior and inferior parts, and decreased thickness of the medial and lateral areas of the hippocampal head and tail for both hemispheres were found for the blind subjects (Figures 3, 5). The increase of the thickness is significant in the hippocampal tail for the left hippocampi, and in the hippocampal head for the right hippocampi (uncorrected). As we all known, the right $\mathrm{HF}$ is related to topographic orientation and spatial memory, whereas the left hippocampus is associated with episodic and autographical event memory (Berthoz, 1997; Maguire, 2001; Viard et al., 2007). Enlarged anterior HF has been related to the enhanced navigation ability with congenital blind subjects (Fortin et al., 2008). Similarly, the right para-hippocampus and visual cortex of the trained blind individuals exhibited more activation than the blindfolded sighted controls during the route recognition task (Kupers et al., 2010). The expansion of superior and inferior regions of the hippocampal head in the current research indicate that the blind individuals may produce more "place cells" to improve the navigation ability (Save et al., 1998; Wolbers and Hegarty, 2010; Lisman et al., 2017). However, only the left hippocampi show significant increase of the thickness after FDR correction in the current research (Figures 4, 6). The left HF mediates learning by binding different sensory modalities, such as the sound and distance information (Gabrieli et al., 1997; Gonzalo et al., 2000; Stark and Squire, 2001; Small, 2002; Norman and O'Reilly, 2003; Preston and Eichenbaum, 2013). The left HF has also been involved in the integration of auditory-visual stimuli (Calvert et al., 2001). The significant increase of the left $\mathrm{HF}$ in the current study indicates that the blindness may prompt the plastic of left hippocampi to acquire the non-spatial signal to improve the navigation ability (Chan et al., 2012).

Previous study found that the congenital blind individuals performed better than the late blind and the sighted blindfolded groups (Passini et al., 1990). Compared to the late blind individuals, enhanced odorant localization abilities was also observed with congenitally blind subjects (Manescu et al., 2021). However, the alterations of the congenital and late blind individuals are nearly the same, and the right hippocampi exhibit more areas with the increased thickness in the current research (Figures 3, 5, $t$-value maps). One possibility is the hippocampal plastic is a long-time course and follows the "Law of Use and Disuse" features of the human organs. The regions associated with straightforward navigation ability (right hippocampi) of the congenital blind subjects may be altered with the stimuli of diverse sensory except visual cues, and the alteration may be inadequate as compared to the adventitiously totally blind individuals. There may be a sharp alteration of the HF with the late blind individuals to deal with the deficiency of visual cues. Another possibility is the relatively small sample size used in the current research, which may be insufficient to detect the detailed difference between the congenital and late blind subjects.

This study has several limitations. The uneven gender distribution with more male subjects in either the congenital blind group or the late blind group may introduce some unexpected influence on the statistical results. This is why we did not consider the gender difference in the current research. In addition, the onset time point of the blindness is with a large span for the late blind subjects, which may also influence the statistical results. It is well known that the alteration of the brain regions may last an extremely long time to be detectable. Late blind subjects with diverse time of duration of the blindness may exhibit different alteration patterns of the hippocampal morphometry. Recruiting the subjects with nearly the same onset time point of the blindness is therefore essential to delineate the features of the hippocampal plastic. On the other hand, there are few studies to conduct longitudinal analysis on the blind HF. To further discover the underlying mechanism of the morphometry alterations of the blind HF, more samples should be recruited and longitudinal analysis based on the follow up scans should also be considered in the future studies.

\section{CONCLUSION}

Current study elucidated the morphometry difference between the blind individuals and sighted controls based on the volume and shape analysis methods. Significant increases of the hippocampal tails for the left hippocampi and hippocampal head for the right hippocampi were observed with the blind individuals compared to the control group. Current results indicate that the plastic of the HF is heterogenous and may occur in distinct subregions of the left and right HF to maintain the navigation ability without the stimuli of visual cues.

\section{DATA AVAILABILITY STATEMENT}

The raw data supporting the conclusion of this article will be made available by the authors, without undue reservation.

\section{ETHICS STATEMENT}

The studies involving human participants were reviewed and approved by Institutional Review Board of Binzhou Medical University. The patients/participants provided their written informed consent to participate in this study.

\section{AUTHOR CONTRIBUTIONS}

NP: drafting the manuscript, analysis and interpretation of data. KZ: data analysis. YaZ: revising the manuscript critically for important intellectual content. DZ: review of manuscript. CD: analysis and interpretation of data. JX: statistical analysis. XL: acquisition of data. YuZ: study conception and obtain funding, design of study, and review of manuscript. All authors contributed to the article and approved the submitted version. 


\section{FUNDING}

This work was supported by the National Natural Science Foundation of China (No. 81801776), Natural Science Foundation of Jiangsu Province (No. BK20170256), and Science and Technology Development Program of Xuzhou (No. KC17164). The work of YaZ was

\section{REFERENCES}

Aanes, S., Bjuland, K. J., Sripada, K., Sølsnes, A. E., Grunewaldt, K. H., Håberg, A., et al. (2019). Reduced hippocampal subfield volumes and memory function in school-aged children born preterm with very low birthweight (VLBW). Neuroimage Clin. 23:101857. doi: 10.1016/j.nicl.2019.101857

Adler, D. H., Wisse, L. E., Ittyerah, R., Pluta, J. B., Ding, S.-L., Xie, L., et al. (2018). Characterizing the human hippocampus in aging and Alzheimer's disease using a computational atlas derived from ex vivo MRI and histology. Proc. Natl. Acad. Sci. 115, 4252-4257. doi: 10.1073/pnas.1801093115

Alme, C. B., Miao, C., Jezek, K., Treves, A., Moser, E. I., and Moser, M.-B. (2014). Place cells in the hippocampus: eleven maps for eleven rooms. Proc. Natl. Acad. Sci. 111, 18428-18435. doi: 10.1073/pnas.1421056111

Arno, P., De Volder, A. G., Vanlierde, A., Wanet-Defalque, M.-C., Streel, E., Robert, A., et al. (2001). Occipital activation by pattern recognition in the early blind using auditory substitution for vision. Neuroimage 13, 632-645. doi: 10.1006/ nimg.2000.0731

Avants, B. B., Tustison, N., and Song, G. (2009). Advanced normalization tools (ANTS). Insight J. 2, 1-35.

Berthoz, A. (1997). Parietal and hippocampal contribution to topokinetic and topographic memory. Philos. Transac. R. Soc. Lond. Ser. B Biol. Sci. 352, 1437-1448. doi: 10.1098/rstb.1997.0130

Calvert, G. A., Hansen, P. C., Iversen, S. D., and Brammer, M. J. (2001). Detection of audio-visual integration sites in humans by application of electrophysiological criteria to the BOLD effect. Neuroimage 14, 427-438. doi: 10.1006/nimg.2001. 0812

Chan, C. C., Wong, A. W., Ting, K. H., Whitfield-Gabrieli, S., He, J., and Lee, T. M. (2012). Cross auditory-spatial learning in early-blind individuals. Hum. Brain Mapp. 33, 2714-2727.

Chebat, D.-R., Chen, J.-K., Schneider, F., Ptito, A., Kupers, R., and Ptito, M. (2007). Alterations in right posterior hippocampus in early blind individuals. Neuroreport 18, 329-333. doi: 10.1097/WNR.0b013e32802b70f8

Chebat, D.-R., Harrar, V., Kupers, R., Maidenbaum, S., Amedi, A., and Ptito, M. (2018). "Sensory substitution and the neural correlates of navigation in blindness" in Mobility of Visually Impaired People. (Eds) E. Pissaloux., R. Velazquez. (Germany: Springer). 167-200.

Chebat, D.-R., Schneider, F. C., Kupers, R., and Ptito, M. (2011). Navigation with a sensory substitution device in congenitally blind individuals. Neuroreport 22, 342-347.

Chen, L. W., Sun, D., Davis, S. L., Haswell, C. C., Dennis, E. L., Swanson, C. A., et al. (2018). Smaller hippocampal CA1 subfield volume in posttraumatic stress disorder. Depress. Anxiety 35, 1018-1029.

Clark, I. A., Monk, A. M., Hotchin, V., Pizzamiglio, G., Liefgreen, A., Callaghan, M. F., et al. (2020). Does hippocampal volume explain performance differences on hippocampal-dependant tasks? Neuroimage 221:117211. doi: 10.1016/j. neuroimage.2020.117211

Collignon, O., Lassonde, M., Lepore, F., Bastien, D., and Veraart, C. (2007). Functional cerebral reorganization for auditory spatial processing and auditory substitution of vision in early blind subjects. Cereb. Cortex 17, 457-465. doi: 10.1093/cercor/bhj162

Crottaz-Herbette, S., Lau, K., Glover, G., and Menon, V. (2005). Hippocampal involvement in detection of deviant auditory and visual stimuli. Hippocampus 15, 132-139. doi: 10.1002/hipo.20039

Despotović, I., Goossens, B., and Philips, W. (2015). MRI segmentation of the human brain: challenges, methods, and applications. Comput. Mathemat. Methods Med. 2015, 1-3. doi: 10.1155/2015/450341 supported by the China Postdoctoral Foundation (No. 2017M612335). The work of JX was supported by the Project funded by China: Postdoctoral Science Foundation (2020M680905). The work of XL was supported by the Shandong Provincial Key Research and Development Project (Grant Nos. 2018GSF118156 and 2018YFJH0501).

Dufour, A., Després, O., and Candas, V. (2005). Enhanced sensitivity to echo cues in blind subjects. Exp. Brain Res. 165, 515-519. doi: 10.1007/s00221-0052329-3

Eichenbaum, H., Dudchenko, P., Wood, E., Shapiro, M., and Tanila, H. (1999). The hippocampus, memory, and place cells: is it spatial memory or a memory space? Neuron 23, 209-226.

Fortin, M., Voss, P., Lord, C., Lassonde, M., Pruessner, J., Saint-Amour, D., et al. (2008). Wayfinding in the blind: larger hippocampal volume and supranormal spatial navigation. Brain 131, 2995-3005. doi: 10.1093/brain/ awn 250

Gabrieli, J. D., Brewer, J. B., Desmond, J. E., and Glover, G. H. (1997). Separate neural bases of two fundamental memory processes in the human medial temporal lobe. Science 276, 264-266. doi: 10.1126/science.276.5310.264

Gagnon, L., Kupers, R., Schneider, F. C., and Ptito, M. (2010). Tactile maze solving in congenitally blind individuals. Neuroreport 21, 989-992.

Gagnon, L., Schneider, F. C., Siebner, H. R., Paulson, O. B., Kupers, R., and Ptito, M. (2012). Activation of the hippocampal complex during tactile maze solving in congenitally blind subjects. Neuropsychologia 50, 1663-1671. doi: 10.1016/j. neuropsychologia.2012.03.022

Gahm, J. K., and Shi, Y. (2016). "Riemannian metric optimization for connectivitydriven surface mapping" in International Conference on Medical Image Computing and Computer-Assisted Intervention. (Germany: Springer). 228-236. doi: 10.1007/978-3-319-46720-7_27

Ge, X., Shi, Y., Li, J., Zhang, Z., Lin, X., Zhan, J., et al. (2015). Development of the human fetal hippocampal formation during early second trimester. Neuroimage $119,33-43$.

Giudice, N. A. (2018). "Navigating Without Vision: principles of Blind Spatial Cognition" in Handbook of Behavioral and Cognitive Geography. (Ed) D. R. Montello. (United Kingdom: Edward Elgar Publishing).

Gonzalo, D., Shallice, T., and Dolan, R. (2000). Time-dependent changes in learning audiovisual associations: a single-trial fMRI study. Neuroimage 11, 243-255. doi: 10.1006/nimg.2000.0540

Jenkinson, M., Beckmann, C. F., Behrens, T. E., Woolrich, M. W., and Smith, S. M. (2012). Fsl. Neuroimage 62, 782-790.

Konrad, C., Ukas, T., Nebel, C., Arolt, V., Toga, A. W., and Narr, K. (2009). Defining the human hippocampus in cerebral magnetic resonance imagesan overview of current segmentation protocols. Neuroimage 47, 1185-1195. doi: 10.1016/j.neuroimage.2009.05.019

Krogsrud, S. K., Tamnes, C. K., Fjell, A. M., Amlien, I., Grydeland, H., and Sulutvedt, U. (2014). Development of hippocampal subfield volumes from 4 to 22 years. Hum. Brain Mapp. 35, 5646-5657.

Kupers, R., and Ptito, M. (2014). Compensatory plasticity and cross-modal reorganization following early visual deprivation. Neurosci. Biobehav. Rev. 41, 36-52.

Kupers, R., Chebat, D. R., Madsen, K. H., Paulson, O. B., and Ptito, M. (2010). Neural correlates of virtual route recognition in congenital blindness. Proc. Natl. Acad. Sci. 107, 12716-12721. doi: 10.1073/pnas.1006199107

Lackner, J. R., and DiZio, P. (2005). Vestibular, proprioceptive, and haptic contributions to spatial orientation. Annu. Rev. Psychol. 56, 115-147. doi: 10.1146/annurev.psych.55.090902.142023

Lai, R., Shi, Y., Dinov, I., Chan, T. F., and Toga, A. W. (2009). "Laplace-Beltrami nodal counts: A new signature for 3D shape analysis" in 2009 IEEE International Symposium on Biomedical Imaging: From Nano to Macro. (United States: IEEE), 694-697. doi: 10.1109/ISBI.2009.5193142

Lazzouni, L., and Lepore, F. (2014). Compensatory plasticity: time matters. Front. Hum. Neurosci. 8:340. doi: 10.3389/fnhum.2014.00340 
Leporé, N., Shi, Y., Lepore, F., Fortin, M., Voss, P., Chou, Y.-Y., et al. (2009). Pattern of hippocampal shape and volume differences in blind subjects. Neuroimage 46, 949-957.

Li, X., Wang, A., Xu, J., Sun, Z., Xia, J., Wang, P., et al. (2019). Reduced Dynamic Interactions Within Intrinsic Functional Brain Networks in Early Blind Patients. Front. Neurosci. 13:268. doi: 10.3389/fnins.2019.00268

Libby, L. A., Ekstrom, A. D., Ragland, J. D., and Ranganath, C. (2012). Differential connectivity of perirhinal and parahippocampal cortices within human hippocampal subregions revealed by high-resolution functional imaging. J. Neurosci. 32, 6550-6560. doi: 10.1523/JNEUROSCI.3711-11.2012

Lisman, J., Buzsáki, G., Eichenbaum, H., Nadel, L., Ranganath, C., and Redish, A. D. (2017). Viewpoints: how the hippocampus contributes to memory, navigation and cognition. Nat. Neurosci. 20, 1434-1447.

Loomis, J. M., Klatzky, R. L., and Golledge, R. G. (2001). Navigating without vision: basic and applied research. Optom. Vis. Sci. 78, 282-289.

Maguire, E. A. (2001). Neuroimaging studies of autobiographical event memory. Philos. Transac. R. Soc. Lond. Ser. B Biol. Sci. 356, 1441-1451.

Maguire, E. A., Gadian, D. G., Johnsrude, I. S., Good, C. D., Ashburner, J., Frackowiak, R. S., et al. (2000). Navigation-related structural change in the hippocampi of taxi drivers. Proc. Natl. Acad. Sci. 97, 4398-4403. doi: 10.1073/ pnas.070039597

Maguire, E. A., Spiers, H. J., Good, C. D., Hartley, T., Frackowiak, R. S., and Burgess, N. (2003). Navigation expertise and the human hippocampus: a structural brain imaging analysis. Hippocampus 13, 250-259.

Manescu, S., Chouinard-Leclaire, C., Collignon, O., Lepore, F., and Frasnelli, J. (2021). Enhanced odorant localization abilities in congenitally blind but not in late-blind individuals. Chemical Senses* 46:bjaa073. doi: 10.1093/chemse/ bjaa073

Muller, R. U., and Kubie, J. L. (1987). The effects of changes in the environment on the spatial firing of hippocampal complex-spike cells. J. Neurosci. 7, 1951-1968.

Naumer, M. J., Doehrmann, O., Müller, N. G., Muckli, L., Kaiser, J., and Hein, G. (2009). Cortical plasticity of audio-visual object representations. Cereb. Cortex 19, 1641-1653.

Ng, B., Toews, M., Durrleman, S., and Shi, Y. (2014). Shape Analysis for Brain Structures. Milwaukee: American Society of Gene and Cell Therapy. 3-49.

Norman, K. A., and O'Reilly, R. C. (2003). Modeling hippocampal and neocortical contributions to recognition memory: a complementary-learning-systems approach. Psychol. Rev. 110:611.

O’Keefe, J., Burgess, N., Donnett, J. G., Jeffery, K. J., and Maguire, E. A. (1998). Place cells, navigational accuracy, and the human hippocampus. Philos. Transac. $R$. Soc. Lond. Ser. B Biol. Sci. 353, 1333-1340. doi: 10.1098/rstb.1998.0287

Passini, R., Proulx, G., and Rainville, C. (1990). The spatio-cognitive abilities of the visually impaired population. Environ. Behav. 22, 91-118.

Preston, A. R., and Eichenbaum, H. (2013). Interplay of hippocampus and prefrontal cortex in memory. Curr. Biol. 23, R764-R773.

Romero, J. E., Coupé, P., and Manjón, J. V. (2017). HIPS: A new hippocampus subfield segmentation method. Neuroimage 163, 286-295.

Saleem, A. B., Diamanti, E. M., Fournier, J., Harris, K. D., and Carandini, M. (2018). Coherent encoding of subjective spatial position in visual cortex and hippocampus. Nature 562, 124-127. doi: 10.1038/s41586-018-0516-1

Save, E., Cressant, A., Thinus-Blanc, C., and Poucet, B. (1998). Spatial firing of hippocampal place cells in blind rats. J. Neurosci. 18, 1818-1826.

Shi, Y., Lai, R., Gill, R., Pelletier, D., Mohr, D., Sicotte, N., et al. (2011). “Conformal metric optimization on surface (CMOS) for deformation and mapping in Laplace-Beltrami embedding space" in International Conference on Medical Image Computing and Computer-Assisted Intervention. (Germany: Springer). 327-334. doi: 10.1007/978-3-642-23629-7_40

Small, S. A. (2002). The longitudinal axis of the hippocampal formation: its anatomy, circuitry, and role in cognitive function. Rev. Neurosci. 13, 183-194.
Stark, C. E., and Squire, L. R. (2001). Simple and associative recognition memory in the hippocampal region. Lear. Mem. 8, 190-197.

Tae, W., Kim, S., Lee, K., Nam, E., Choi, J., and Park, J. (2011). Hippocampal shape deformation in female patients with unremitting major depressive disorder. Am. J. Neuroradiol. 32, 671-676. doi: 10.3174/ajnr.A2367

Viard, A., Piolino, P., Desgranges, B., Chételat, G., Lebreton, K., Landeau, B., et al. (2007). Hippocampal activation for autobiographical memories over the entire lifetime in healthy aged subjects: an fMRI study. Cereb. Cortex 17, 2453-2467. doi: 10.1093/cercor/bhl153

Voss, P., Gougoux, F., Zatorre, R. J., Lassonde, M., and Lepore, F. (2008). Differential occipital responses in early-and late-blind individuals during a sound-source discrimination task. Neuroimage 40, 746-758. doi: 10.1016/j. neuroimage.2007.12.020

Weeks, R., Horwitz, B., Aziz-Sultan, A., Tian, B., Wessinger, C. M., Cohen, L. G., et al. (2000). A positron emission tomographic study of auditory localization in the congenitally blind. J. Neurosci. 20, 2664-2672. doi: 10.1523/JNEUROSCI. 20-07-02664.2000

Weisberg, S. M., Newcombe, N. S., and Chatterjee, A. (2019). Everyday taxi drivers: Do better navigators have larger hippocampi? Cortex 115, 280-293. doi: 10. 1016/j.cortex.2018.12.024

Wolbers, T., and Hegarty, M. (2010). What determines our navigational abilities? Trends Cogn. sci. 14, 138-146.

Woolard, A. A., and Heckers, S. (2012). Anatomical and functional correlates of human hippocampal volume asymmetry. Psychiatry Res. Neuroimaging 201, 48-53.

Worsley, K. J., Taylor, J., Carbonell, F., Chung, M., Duerden, E., Bernhardt, B., et al. (2009). A Matlab toolbox for the statistical analysis of univariate and multivariate surface and volumetric data using linear mixed effects models and random field theory. NeuroImage 47:S102.

Xu, F., Ge, X., Shi, Y., Zhang, Z., Tang, Y., Lin, X., et al. (2020). Morphometric development of the human fetal cerebellum during the early second trimester. Neuroimage 207:116372. doi: 10.1016/j.neuroimage.2019.11 6372

Yushkevich, P. A., Piven, J., Hazlett, H. C., Smith, R. G., Ho, S., Gee, J. C., et al. (2006). User-guided 3D active contour segmentation of anatomical structures: significantly improved efficiency and reliability. Neuroimage 31, 1116-1128. doi: 10.1016/j.neuroimage.2006.01.015

Yushkevich, P. A., Pluta, J. B., Wang, H., Xie, L., Ding, S.-L., Gertje, E. C., et al. (2015). Automated volumetry and regional thickness analysis of hippocampal subfields and medial temporal cortical structures in mild cognitive impairment. Hum. Brain Mapp. 36, 258-287. doi: 10.1002/hbm.22627

Conflict of Interest: The authors declare that the research was conducted in the absence of any commercial or financial relationships that could be construed as a potential conflict of interest.

Publisher's Note: All claims expressed in this article are solely those of the authors and do not necessarily represent those of their affiliated organizations, or those of the publisher, the editors and the reviewers. Any product that may be evaluated in this article, or claim that may be made by its manufacturer, is not guaranteed or endorsed by the publisher.

Copyright (c) 2021 Pan, Zheng, Zhao, Zhang, Dong, Xu, Li and Zheng. This is an open-access article distributed under the terms of the Creative Commons Attribution License (CC BY). The use, distribution or reproduction in other forums is permitted, provided the original author(s) and the copyright owner(s) are credited and that the original publication in this journal is cited, in accordance with accepted academic practice. No use, distribution or reproduction is permitted which does not comply with these terms. 